\title{
Sistema web para gestión de estadías profesionales para TSU e Ingeniería
}

\section{Web System for managing professional stays for TSU and Engineering}

\author{
HERRERA-CRUZ, Joel†*, GALICIA-ESCALANTE, Alejandra y ESQUIVEL-GONZÁLEZ, Jesús
}

Universidad Tecnológica del Valle de Toluca

ID 1 $1^{\text {er }}$ Autor: Joel, Herrera-Cruz / ORC ID: 0000-0003-3237-769X, CVU CONACYT ID: 680821

ID $1^{\text {er }}$ Coautor: Alejandra, Galicia-Escalante /ORC ID: 0000-0003-0688-7522, CVU CONACYT ID: 401191

ID 2 ${ }^{\text {do }}$ Coautor: Jesús, Esquivel-González / ORC ID: 0000-0002-5114-1917, CVU CONACYT ID: 679703

DOI: $10.35429 / J C S .2019 .10 .3 .16 .24$

Recibido: 12 de Agosto, 2019; Aceptado 30 de Diciembre, 2019

\begin{abstract}
Resumen
Las estadías profesionales permiten a los estudiantes poder incorporarse al mundo laboral de una manera rápida y adquirir conocimientos que permitan mejorar su experiencia profesional. Se desarrolló una herramienta de software que permita gestionar el proceso de estadías profesionales de los estudiantes de la carrera de TSU y de Ingeniería de Tecnologías de la Información y Comunicación de la Universidad Tecnológica del Valle de Toluca, el cual permita coadyuvar a mejorar la atención de los asesores académicos hacia los estudiantes para poder llevar el control de: . Evaluaciones 1ra y 2da de estadía por parte de asesor académico y asesor externo -Poder subir a la plataforma en línea las memorias de estadía - Llevar reportes estadísticos sobre formatos oficiales de convenios entre escuela-empresa-estudiante - Estadísticos de las habilidades que desempeñan los estudiantes en las estadías profesionales para poder saber cuál es el campo en donde hay más demanda y la pertinencia tecnológica en los parques industriales, empresas descentralizadas en los diferentes sectores (educativo, público y privado). - Llevar el seguimiento de las actividades que desempeñan los estudiantes en las empresas. Todos los requerimientos anteriores son con el propósito de ver las necesidades de las organizaciones e ir adecuando los conocimientos impartidos en clase para que los estudiantes estén actualizados y cumplan con las expectativas de los empleadores y puedan incorporarse al mundo laboral.
\end{abstract}

\begin{abstract}
Professional stays allow students to enter the workplace quickly and acquire knowledge to improve their professional experience. A software tool was developed that allows to manage the process of professional stays of the students of the TSU career and engineering of Information and Communication Universidad Tecnologica del Valle de Toluca, which allows to help improve the care of the academic advisors towards the students to be able to take control of: . 1st and 2nd stay evaluations by academic advisor and external advisor -To be able to upload the stay memories to the online platform - Keep statistical reports on official formats of agreements between school-company-student - Statisticians of the skills that students perform in professional stays to be able to know what is the field where there is more demand and what technologies are currently being used in the industry. - Keep track of the activities that students perform in companies. All of the above with the purpose of seeing the needs of organizations and adapting the knowledge taught in class so that students remain competitive and can enter the world of work
\end{abstract}

Tool, Analysis, Tracing

Citación: HERRERA-CRUZ, Joel, GALICIA-ESCALANTE, Alejandra y ESQUIVEL-GONZÁLEZ, Jesús. Sistema web para gestión de estadías profesionales para TSU e Ingeniería. Revista de Simulación Computacional. 2019. 3-10: 16-24

\footnotetext{
* Correspondencia al Autor: (joel.herrera@)utvtol.edu.mx)

$\dagger$ Investigador contribuyendo como primer autor.
} 


\section{Introducción}

Las estadías profesionales son el medio que permite a los estudiantes del nivel educativo Técnico Superior Universitario y de Ingeniería el poder adquirir experiencia profesional en el área donde se desea especializar, para muchos es el medio de incorporarse al mundo laboral y así adquirir conocimientos prácticos que fortalezcan sus habilidades, actitudes y conocimientos los cuales les permita competir con cualquier estudiante egresado de otras Instituciones Educativas. El proyecto de Sistema de Estadías desarrollado para la Dirección de Carrera de Tecnologías de la Información y Comunicación (TIC), es un proyecto Web que permitirá gestionar la información y control de las estadías de los programas educativos con los que cuenta la carrera. Las estadías se cursan en el sexto cuatrimestre y onceavo de las carreras de TSU e Ingeniería respectivamente, como parte de su preparación, ya que es bien sabido que es el mejor medio para aplicar todos los conocimientos que se adquirieron durante los anteriores cuatrimestres.

En las estadías profesionales el estudiante adquiere competencias que no adquiere en aulas, permitiéndole integrar diversas áreas de conocimiento participando en proyectos multidisciplinarios en los diferentes sectores, fortaleciendo el trabajo en equipo, trabajo autodidacta, relaciones interpersonales, proactividad entre otras. Con el desarrollo del proyecto se logra agilizar la concentración de la información de los datos de las empresas, datos de los proyectos a desarrollar durante la estadía profesional y datos personales de los estudiantes, permitiendo la generación de documentos oficiales del sistema de calidad y reportes solicitados.

\section{Justificación}

En el reglamento de la Universidad Tecnológica del Valle de Toluca se menciona que los estudiantes deberán cursar 2 periodos de estadía en la formación de su ingeniería, un periodo en 6 to cuatrimestre y el otro en 11vo cuatrimestre, en periodos de tiempo de 480 horas las cuales deberán realizarse en organismos privados, públicos o gubernamentales, y se deberá realizar un proyecto adecuado a su formación profesional donde aplique todos los conocimientos adquiridos en cuatrimestres anteriores.
En este proceso de estadía el estudiante tendrá en todo momento 2 asesores, el EXTERNO el cual es la persona asignada por la organización la cual lo estará guiando en como ir desarrollando dicho proyecto, indicando avances, modificación de requerimientos, adecuación a las nuevas exigencias de lo que se pretende automatizar y/o desarrollar, con esta persona trabaja directamente en la institución que solicita el proyecto $\mathrm{y}$ el asesor ACADÉMICO es un profesor asociado $\mathrm{C}$ de la UTVT que fungirá como asesor técnico como apoyo para el desarrollo del proyecto de la empresa y el que también llevará el seguimiento de su memoria de estadía. El estudiante tendrá 2 evaluaciones, una a la mitad de su estadía y la otra al final, en la cual se evalúa tanto conocimientos, actitud y desarrollo del proyecto asignado. El promedio de estas 2 evaluaciones deberá ser igual o mayor a 8.0 para poder aprobar la estadía. Al final el estudiante es aquí donde le deberá entregar un documento tipo tesina que se le nombra Memoria de Estadía a su asesor académico, en la cual redacta a detalle el marco teórico, metodología de desarrollo, desarrollo y resultados de las actividades y proyecto(s) realizados en su estadía.

Esto ha generado satisfacciones al estudiante, empresarios y docentes, los cuales han visto a lo largo de los años un impacto positivo en la zona de influencia de la UTVT. Ya que los proyectos que se han realizado de estadía han permitido implementar soluciones en distintas áreas de las organizaciones lo cual permite que los estudiantes puedan quedarse a trabajar en dichas organizaciones al finalizar su periodo de estadía, adquiriendo experiencia laboral y cambiar su visión sobre las tendencias actuales en el área de las TIC para mantenerse actualizados y competitivos.

\section{Problemática}

Actualmente el proceso de definir o asignar las estadías profesionales, si bien se lleva acabo pero no de la manera más eficiente, debido a que no se tiene un historial sobre las necesidades que ha presentado cada empresa y de los proyectos que oferta a los estudiantes, provocando que algunas veces el estudiante no está especializado totalmente en lo que requiere la empresa, provocando que algunas veces los proyectos que se desarrollaron en la estadía no cumplen con las expectativas de los Asesores Externos y que los proyectos desarrollados no se implementen en la empresa.

HERRERA-CRUZ, Joel, GALICIA-ESCALANTE, Alejandra ESQUIVEL-GONZÁLEZ, Jesús. Sistema web para gestión de estadías profesionales para TSU e Ingeniería. Revista de Simulación Computacional. 2019 
Así mismo se necesita saber de una manera más puntual las tendencias de tecnología que actualmente se necesitan en las organizaciones para que los profesores de la UTVT en específico de la dirección de TIC puedan adecuar los contenidos en las hojas de asignatura a fin de estar mas actualizados para poder tener estudiantes más competitivos.

Este trabajo describe el desarrollo de un proyecto de software web que permita llevar el seguimiento de los estudiantes que están en estadía, poder llevar sus evaluaciones y memorias de estadía, pero sobre todo poder registrar por cada estadía que área de conocimiento de las TIC se aplicó en el proyecto, que tipo de Hardware o Software se utilizo en el proyecto, Observaciones generales de la evaluación de estudiantes y recomendaciones de las organizaciones sobre tecnología emergente que a los profesores les permita saber las tendencias de la industria para poder impartirla en clases y poder mantenerse actualizado.

\section{Hipótesis}

La hipótesis contempla que la implementación de un sistema de software web donde pueda capturarse el detalle de cada estadía y que permita mediante el histórico de ellas saber la tendencia en tecnología de Software, Hardware, Metodologías, Redes, Lenguajes de Programación, Inteligencia de Negocios, etc. que requiere una organización y esto permita poder asignar de una manera mas eficiente a los estudiantes que cubran el perfil solicitado para cada estadía y así poder crear proyectos eficaces y eficientes para las organizaciones y que los estudiantes puedan fortalecer aún más sus habilidades en el área de conocimiento en la que se están especializando

\section{Objetivo general}

Diseñar, desarrollar e implementar un sistema web en la Dirección de TIC de la UTVT que permita gestionar la información de las estadías de estudiantes de 6to y 11vo cuatrimestre y de esta manera poder asignar de una manera mas eficiente a estudiantes a los proyectos de Estadías Profesionales tomando en cuenta los alcances de los proyectos y los conocimientos y habilidades de los estudiantes

\section{Objetivos Específicos}

- Especificar la metodología para el diseño de la plataforma.

- Establecer los roles que tendrá cada usuario (estudiantes, asesores académicos, asesores externos) dentro de la plataforma.

- $\quad$ Diseñar y programar la interfaz de cada usuario

- Capturar la información de las estadías profesionales para poder generar los reportes que permitan la toma de decisiones al asignar estudiantes a estadías

Evaluar el impacto de la plataforma tanto en docentes, como en estudiantes durante el tiempo de la implementación.

- Tener una administración más precisa de los resultados en cada ciclo de estadías y permita apoyar a la toma de decisiones por parte del tutor de grupo quien es el encargado de la asignación de organización y asesores académicos tomando en cuenta las fortalezas de este último.

\section{Marco Teórico}

\section{Metodología desarrollo de SW}

El modelo en cascada es un proceso de desarrollo secuencial, en el que el desarrollo de software se concibe como un conjunto de etapas que se ejecutan una tras otra. Se le denomina así por las posiciones que ocupan las diferentes fases que componen el proyecto, colocadas una encima de otra, y siguiendo un flujo de ejecución de arriba hacia abajo, como una cascada.

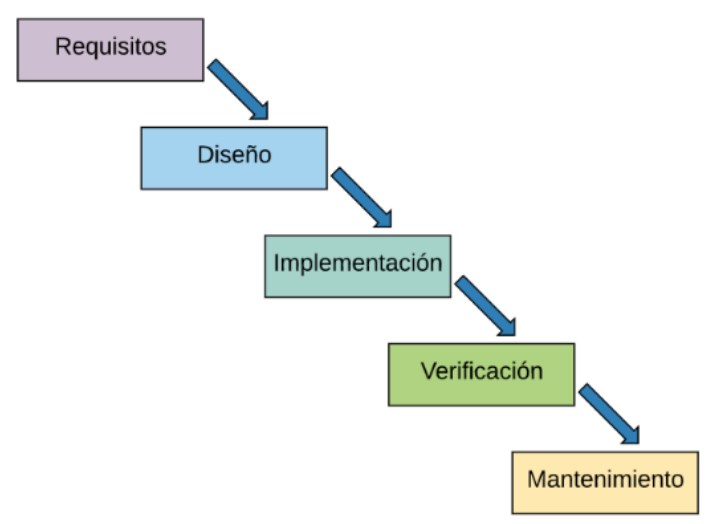

Figura 1 Fases de la metodología cascada 


\section{Fases del modelo}

El modelo de desarrollo en cascada sigue una serie de etapas de forma sucesiva, la etapa siguiente empieza cuando termina la etapa anterior. Las fases que componen el modelo son las siguientes:

\section{a) Requisitos del software}

En esta fase se hace un análisis de las necesidades del cliente para determinar las características del software a desarrollar, y se especifica todo lo que debe hacer el sistema sin entrar en detalles técnicos. Se debe ser especialmente cuidadoso en esta primera fase, ya que en este modelo no se pueden añadir nuevos requisitos en mitad del proceso de desarrollo.

Por lo tanto, esta es la etapa en la que se lleva a cabo una descripción de los requisitos del software, y se acuerda entre el cliente y la empresa desarrolladora lo que el producto deberá hacer. Disponer de una especificación de los requisitos permite estimar de forma rigurosa las necesidades del software antes de su diseño. Además, permite tener una base a partir de la cual estimar el costo del producto, los riesgos y los plazos.

En el documento en el que se especifican los requisitos, se establece una lista de los requerimientos acordados. Los desarrolladores deben comprender de forma clara el producto que van a desarrollar. Esto se consigue teniendo una lista detallada de los requisitos y con una comunicación fluida con el cliente hasta que termine el tiempo de desarrollo.

\section{b) Diseño}

En esta etapa se describe la estructura interna del software, y las relaciones entre las entidades que lo componen. Descompone y organiza el sistema en elementos que puedan elaborarse por separado, aprovechando las ventajas del desarrollo en equipo. Como resultado surge el SDD (Documento de Diseño del Software), que contiene la descripción de la estructura relacional global del sistema y la especificación de lo que debe hacer cada una de sus partes, así como la manera en que se combinan unas con otras.
Es conveniente distinguir entre diseño de alto nivel o arquitectónico y diseño detallado. El primero de ellos tiene como objetivo definir la estructura de la solución (una vez que la fase de análisis ha descrito el problema) identificando grandes módulos (conjuntos de funciones que van a estar asociadas) y sus relaciones. Con ello se define la arquitectura de la solución elegida. El segundo define los algoritmos empleados y la organización del código para comenzar la implementación.

\section{c) Implementación}

En esta fase se programan los requisitos especificados haciendo uso de las estructuras de datos diseñadas en la fase anterior. La programación es el proceso que lleva de la formulación de un problema de computación, a un programa que se ejecute produciendo los pasos necesarios para resolver dicho problema. Al programar, tenemos que realizar actividades como el análisis de las condiciones, la creación de algoritmos, y la implementación de éstos en un lenguaje de programación específico. Un algoritmo es un conjunto de instrucciones o reglas bien definidas y ordenadas que permiten llevar a cabo una actividad mediante pasos sucesivos.

\section{d) Verificación}

Como su propio nombre indica, una vez que se termina la fase de implementación, se verifica que todos los componentes del sistema funcionen correctamente y cumplen con los requisitos. El objetivo de las pruebas es el de obtener información de la calidad del software, y sirven para: encontrar defectos o bugs, aumentar la calidad del software, refinar el código previamente escrito sin miedo a romperlo o introducir nuevos bugs, etc.

\section{e) Instalación y mantenimiento}

Una vez se han desarrollado todas las funcionalidades del software $y$ se ha comprobado que funcionan correctamente, se inicia la fase de instalación y mantenimiento. Se instala la aplicación en el sistema y se comprueba que funcione correctamente en el entorno en que se va a utilizar. A partir de ahora hay que asegurarse de que el software funcione y hay que destinar recursos a mantenerlo. 
El mantenimiento del software consiste en la modificación del producto después de haber sido entregado al cliente, ya sea para corregir errores o para mejorar el rendimiento o las características. El propósito de esta fase es mantener el valor del software a través del tiempo. Esto puede hacerse añadiendo nuevos requisitos, corrigiendo errores, renovando el aspecto visual, mejorando la eficiencia o añadiendo nueva tecnología. El periodo de mantenimiento puede durar años, por lo que es una fase clave del modelo en cascada.

Para llevar a cabo correctamente la fase de mantenimiento, se necesita trazar un plan de antemano que nos prepare para todos los escenarios que puedan producirse durante esta fase. Para evitar futuros conflictos con el cliente, en el plan hay que especificar cómo los usuarios solicitarán las modificaciones o la corrección de errores, hacer una estimación del coste de la modificación de funcionalidades o corrección de errores, quién se encargará del mantenimiento, durante cuánto tiempo se dará soporte al software, etc. Fuente: (Open Classroom, 2015)

\section{Estadía profesional}

La práctica profesional solo puede desarrollarse trabajando, es la experiencia acumulada de situaciones reales que se presentan en la vida real antes que un conocimiento forjado en los libros. Es común señalar que existe un muro entre la educación formal y el universo del trabajo. En las universidades suele fomentarse el aprendizaje de un sin número de disciplinas que muchas veces el estudiante tarda en poner en práctica o se encuentra perplejo a la hora de aplicar a la experiencia cotidiana.

Es por ello que existen formas de lograr un puente entre ambas situaciones, formas que implican un primer aproximamiento mediante prácticas que están escasamente remuneradas. Este tipo de prácticas tienen que ser ricas en lo que respecta a experiencias de formación de tal manera que preparen a los estudiantes para las experiencias reales que puedan experimentar cuando se alejen de un marco educativo que los contiene. Fuente: (Editorial Definición MX. 2014).

\section{Metodología a desarrollar}

\section{Tipo de Investigación}

Investigación Aplicada

\section{Cronograma de Actividades:}

\begin{tabular}{|c|c|c|}
\hline Actividades & $\begin{array}{l}\text { Fase } 1 \\
\text { May-Ago } \\
2019\end{array}$ & $\begin{array}{c}\text { Fase } 2 \\
\text { Sep.- Dic } \\
2019\end{array}$ \\
\hline $\begin{array}{lrr}\text { Análisis y } & \text { diseño del sistema } \\
\text { con base en los formatos } \\
\text { utilizados en el } & \text { SGI } \\
\text { correspondientes } & & \text { al } \\
\text { procedimiento de estadías } & \\
\end{array}$ & & \\
\hline $\begin{array}{l}\text { Desarrollo de la aplicación y sus } \\
\text { diferentes módulos }\end{array}$ & & \\
\hline $\begin{array}{l}\text { Captura de la información de } \\
\text { estadía de estudiantes de 11vo } \\
\text { cuatrimestre }\end{array}$ & & \\
\hline $\begin{array}{l}\text { Generación de pruebas y } \\
\text { reportes adecuados al SGI }\end{array}$ & & \\
\hline $\begin{array}{l}\text { Modificar la base de datos } \\
\text { anexando los nuevos campos } \\
\text { para tener información mas } \\
\text { precisa de todos los } \\
\text { involucrados en el proceso de } \\
\text { estadía. }\end{array}$ & & \\
\hline $\begin{array}{llr}\text { Adecuación a los módulos } \\
\text { programados } & \text { para } & \text { una } \\
\text { asignación mas eficaz de } & \text { des } \\
\text { estudiantes a estadías } & \end{array}$ & & \\
\hline
\end{tabular}

\section{Métodos Teóricos}

Las etapas de la metodología cascada aplicadas al proyecto de seguimiento de estadías son los siguientes:

a) Requisitos del software: Conocer el procedimiento de estadías profesionales de la UTVT el cual esta descrito en el Procedimiento P-EDU-03 Rev. 4 del sistema del SGI y analizar el proceso de seguimiento de estadía que debe de seguir el estudiante, empresa y asesores, para que la logística del sistema vaya a la par del SGI, así como concentrar la evidencia de toda la documentación que se necesita para evidenciar el proceso.

Se analizo toda la información que se requiere para la asignación de estadía, así como la documentación que es necesaria en el proceso de estadía. Para este proceso se ocupó el formato del SGI R-EDU-61 Proyectos de estadía 


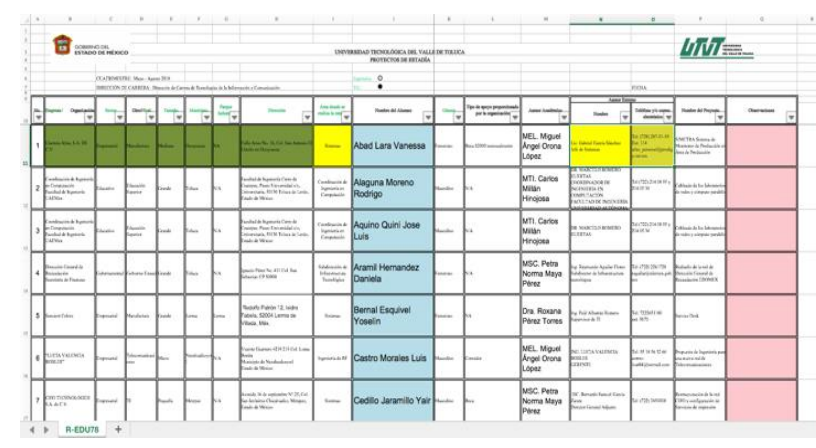

Figura 2 Información de proyectos de estadía profesional

- $\quad$ R-EDU-59 Carta de Estadía Profesional

- R-EDU-44 Encuesta Final de Estadía Profesional TSU-TIC-SI

- R-EDU-49 Encuesta Final de Estadía Profesional TSU-TIC_RT

R-EDU-51 Encuesta Final de Estadía Profesional ITIC

R-EDU-63 Evaluación de Estadía

- $\quad$ R-EDU-62 Reporte de visitas de asesoría a estudiantes en Estadía

b) Diseño: En esta fase se realizarán los Mokups de las interfaces web del sistema, así como adecuar las interfaces a la base de datos, arquitectura de programación (Modelo Vista Controlador), el cual permita un diseño intuitivo con una buena experiencia de usuario. Se creo el diagrama relacional de la base de datos que abarcara todos los módulos a programar de la fase 1 del sistema

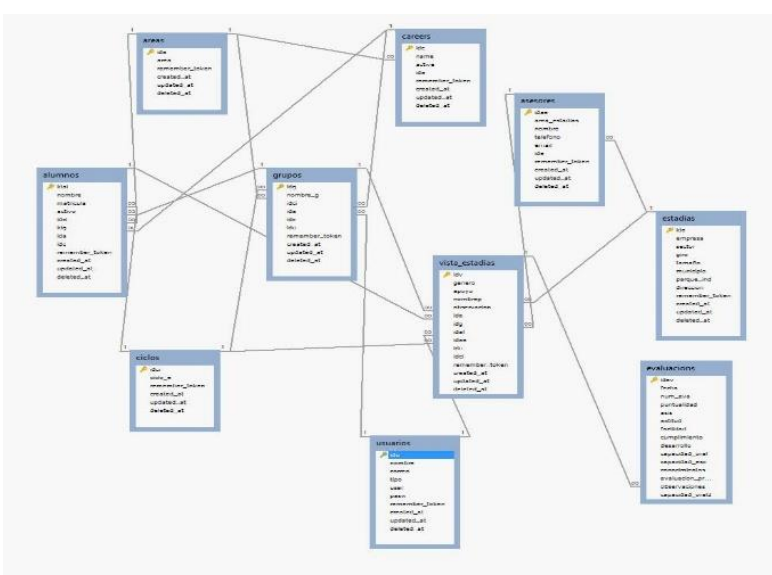

Figura 3 Diagrama Relacional base de datos

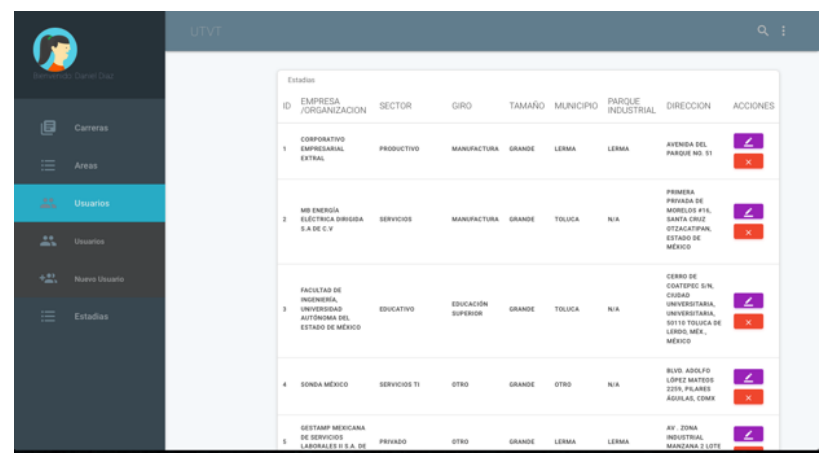

Figura 4 Creación de diseño final para interfaces de usuario y estandarización de vistas

Se crea un nuevo instrumento de captura de información de proyectos de estadía que permita evaluar los tipos de proyecto, tecnologías emergentes de las empresas y caracterización de las habilidades de los estudiantes

c) Implementación: Se procede a realizar la programación del sistema web, ocupando como lenguaje de programación PHP en su Framework Laravel 5.8 como backend y Java, JS, AJAX y Json como Frontend, base de datos Mysql 5.4, Diseño de interface Bootstrap, HTML 5 y css 3, en donde la programación se realizará modular y escalar. La programación será controlada por la herramienta GIT para registro de versiones y así a futuro pueda darse un mantenimiento más eficaz a la aplicación

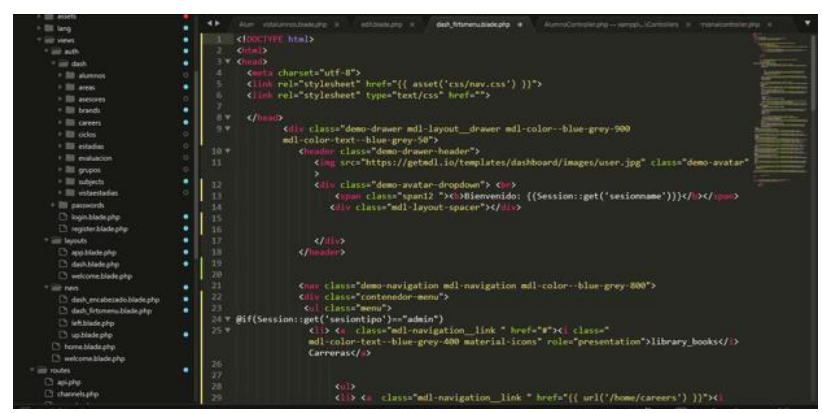

Figura 5 Codificación del sistema en arquitectura MVC

A continuación, se describen las interfaces ya programadas de la primera fase de desarrollo del sistema

Una vez terminada la interfaz de todo el sistema, se empezaron a crear los formularios que pudimos rescatar en el análisis, se crearon los modelos, las vistas y los controladores que fungieron como la arquitectura principal del proyecto. Por mencionar algunas de las vistas mas importantes se destacan: 
El formulario donde se dan de alta las carreras que pertenecen a la Dirección de Carrera de Tecnologías de la Información y Comunicación

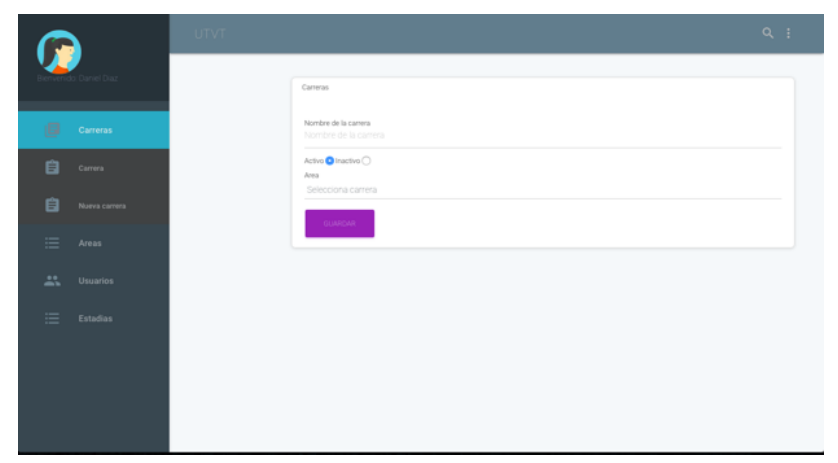

Figura 6 Formulario de carreras

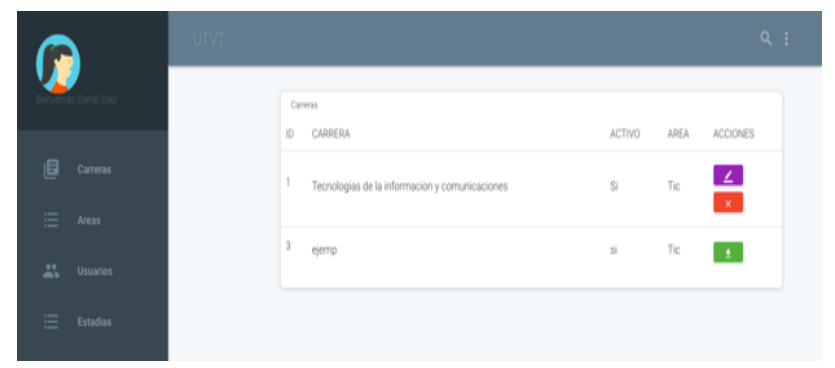

Figura 7 Índex de Carrera

La carrera cuenta con su formulario y su índex esto se creó para tener un mayor control de los estudiantes y saber en qué carrera y área están inscritos. Se cuenta con un formulario para crear usuarios que tendrán acceso a la plataforma en este caso serán los asesores, estudiantes y tutores responsables de estadías profesionales

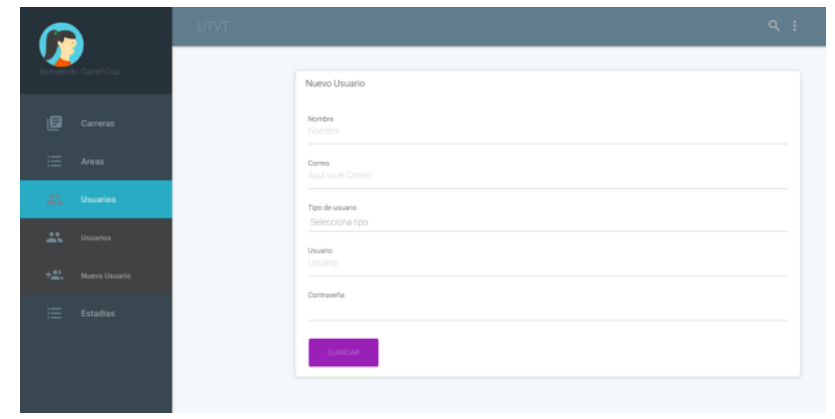

Figura 1 Formulario de usuario

Para agilizar la carga de los estudiantes se desarrolló en una programación de carga de archivos de CSV, en el cual ya teniendo la lista de grupos oficial en Excel estos pueden ser cargados al sistema de forma automática para poder agilizar el proceso de captura de estudiantes, en esta carga se asignan automáticamente estudiantes a grupos, ciclos escolar y área de carrera

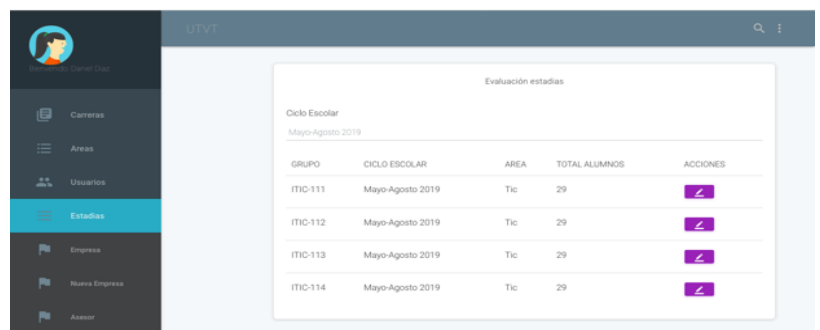

Figura 9 Cargar de estudiantes al sistema CSV

Este es el apartado más importante de todo el sistema donde se hace el registro de toda la información acerca de la empresa y del estudiante al igual que la evaluación de las estadías, esta adecuado a que cumpla con todos los requisitos que pide el formato R-EDU-61, el cual concentra toda la información del proyecto estadía, tipo de proyecto a realizar, nombres de los asesores externos y académicos, detalles de la empresa, contactos con la empresa, los cuales servirán como guía para la información y formatos que se expiden durante el desarrollo de la estadía

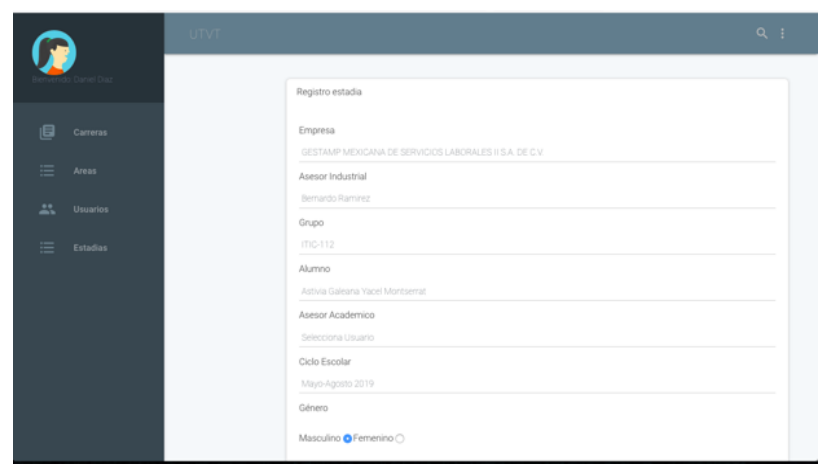

Figura 10 Formulario de alta de estadías profesionales

Después de haber capturado el sistema de estadías los asesores podrán en su sesión ver que estudiantes tienen asignados a su cargo y poder conocer el detalle de todo su proyecto de estadía profesional. La cual podrá ser editada durante todo el proceso de la estadía profesional por el asesor académico y el tutor de estadías. Esta información podrá exportarse a Excel en el formato R-EDU-61 del SGI.

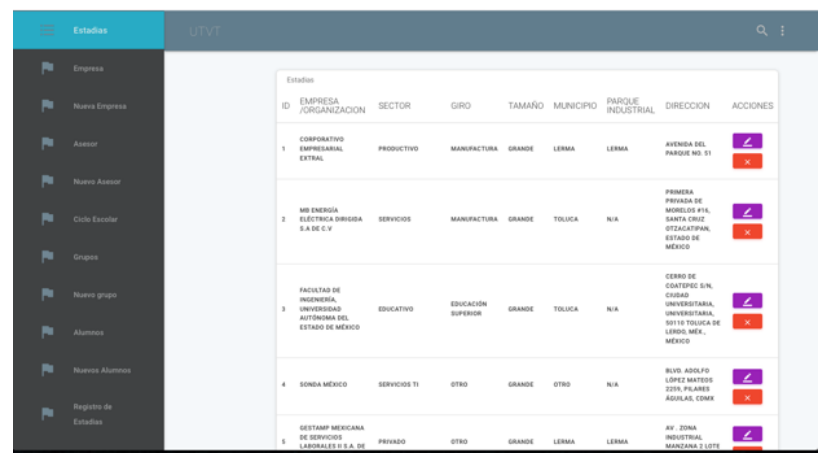

Figura 11 Interfaz de Reporte de estadías

HERRERA-CRUZ, Joel, GALICIA-ESCALANTE, Alejandra y ESQUIVEL-GONZÁLEZ, Jesús. Sistema web para gestión de estadías profesionales para TSU e Ingeniería. Revista de Simulación Computacional. 2019 
Se desarrollo el modulo de captura de evaluaciones la cual se adecuo al formato de calidad vigente R-EDU-63, en el cual el asesor académico podrá capturar las evaluaciones 1 y 2 de la estadía, así como las observaciones de seguimiento dadas por los asesores externos. Esta información se podrá exportar a Excel en el formato de calidad de evaluación de estadía

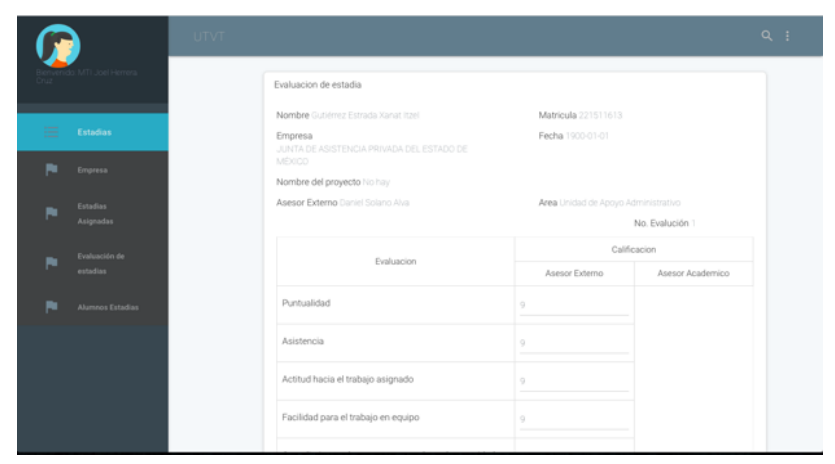

Figura 12 Formulario de alta de calificaciones para la 1ra y 2da evaluación

En todo momento el asesor académico podrá tener un resumen de las calificaciones de los estudiantes evaluación, así como el sistema automáticamente ira calculando los promedios de las evaluaciones individuales, así como el promedio general del cuatrimestre

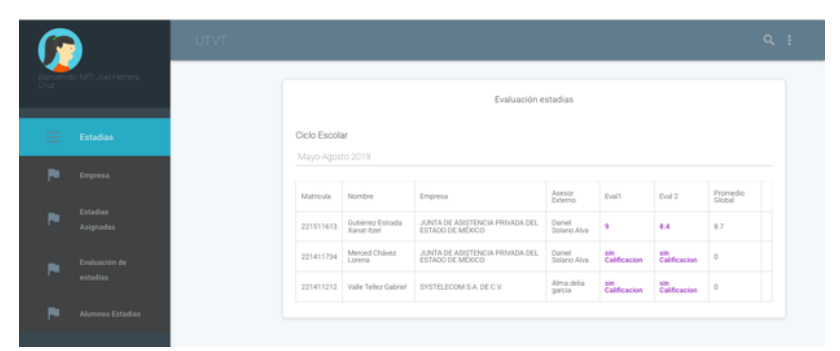

Figura 13 Reporte de calificaciones de estudiantes asignados

c) Verificación: Se hicieron pruebas son la generación de estudiantes de estadía del cuatrimestre Mayo - Agosto del 2019, y se dieron de alta todos los estudiantes, sus proyectos y sus evaluaciones, para garantizar que el funcionamiento del sistema es correcto

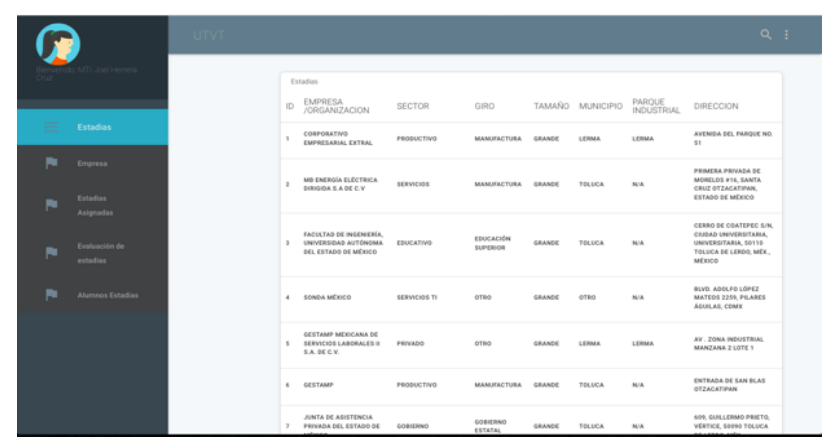

Figura 14 Verificación de uso de sistema

ISSN 2523-6865

ECORFAN® Todos los derechos reservado d) Pruebas y mantenimiento: En esta fase, se hicieron pruebas de concurrencia para verificar que varios usuarios logueados al mismo tiempo no tuvieran problemas de conectividad o duplicidad de información al registro de las evaluaciones, se verifico que la base de datos todos los campos estuvieran totalmente llenos y no viajara información que no tuviera que ver con los campos. Se generaron los reportes del sistema que fueran congruentes a los formatos que se manejan en el SGI

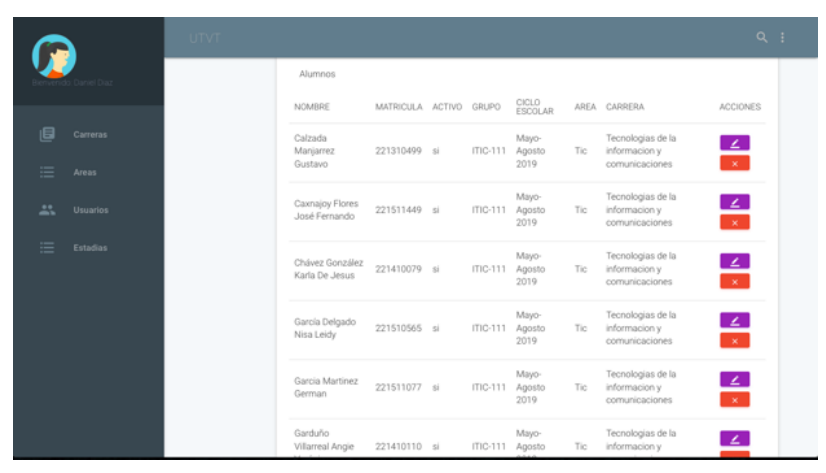

Figura 15 Registro de estudiantes guardados

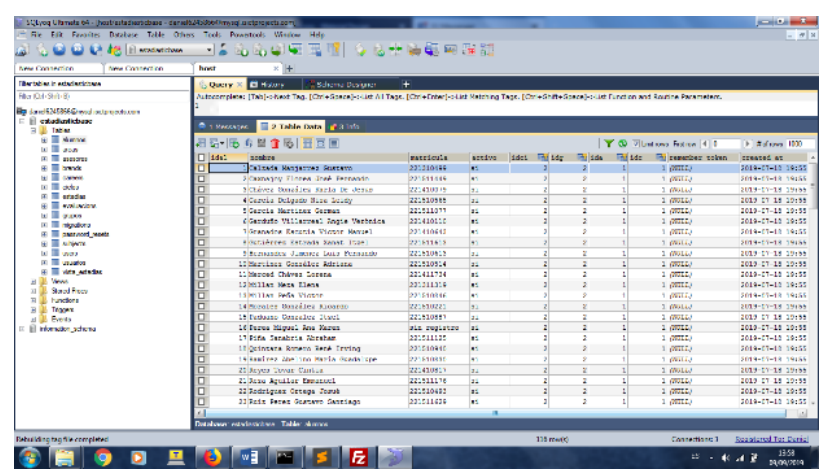

Figura 16 Pruebas de base de datos

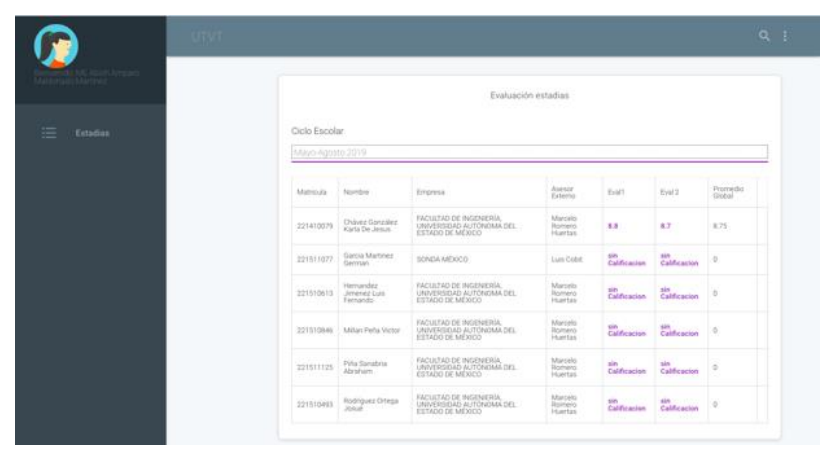

Figura 17 Pruebas en interfaces de asesores académicos 


\section{Resultados}

- $\quad$ Se implementó la fase 1 del sistema de gestión de estadías profesionales.

Se dieron de alta para las pruebas todos los 116 estudiantes que hicieron su estadía en 11vo cuatrimestre de ingeniería, los cuales fueron clasificados en sus grupos y programas educativos, así como las empresas y sus representantes como asesores industriales

- Asignación de estadías a los asesores académicos y se registraron los proyectos de estadías profesionales para generar el documento R-EDU-61 proyectos de estadía del SGI

Se dieron de alta las evaluaciones de los estudiantes de la 1ra y 2 da evaluación de estadía.

\section{Agradecimiento}

El trabajo fue apoyado por la Dirección de Tecnologías de la Información y Comunicación y Secretaría Académica de la Universidad Tecnológica del Valle de Toluca.

\section{Conclusiones}

El sistema fue realizado en una primera etapa para llevar el control de las empresas participantes y sus colaboradores en los proyectos de estadía, con el fin de llevar una administración mas clara de toda la información necesaria para llenar los formatos que indica el procedimiento de estadía marcado en el Sistema de Gestión Integral Institucional.

Esta primera etapa se concluye con la automatización de todo lo solicitado en cada uno de los formatos utilizados en periodos anteriores los cuales eran llenados a mano y sin tener una idea clara de toda la información que se maneja.

\section{Referencias}

Editorial Definición MX. (2014). Disponible http://www.revistavirtualpro.com/biblioteca/am bientes-virtuales-de-aprendizaje-unametodologia-para-su-creacion. Consultado 10 de agosto de 2019
Open Classroom (2015). Disponible https://openclassrooms.com/en/courses/430915 1-gestiona-tu-proyecto-de-desarrollo/4538221en-que-consiste-el-modelo-en-cascada.

Consultado 5 de mayo de 2019

Roger S. Pressman. (2002). Ingeniería de software un enfoque práctico. (pp. 259-271). Madrid: McGraw Hill. 